\title{
Metabolomics analysis of herb-partitioned moxibustion treatment on rats with diarrhea-predominant irritable bowel syndrome
}

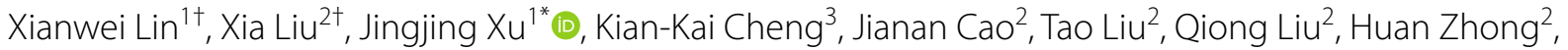 \\ Guiping Shen ${ }^{1}$, Jiyang Dong ${ }^{1}$ and Xiaorong Chang $^{2^{*}}$
}

\begin{abstract}
Background: Irritable bowel syndrome (IBS) is a common functional gastrointestinal disorder, which is commonly treated with antidiarrhoeal, antispasmodics, serotonergic agents or laxative agents. These treatments provide relief for IBS symptoms but may also lead to undesired side effects. Previously, herb-partitioned moxibustion (HPM) treatment has been demonstrated to be effective in ameliorating symptoms of IBS. However, the underlying mechanism of this beneficial treatment is yet to be established. The aim of the current study was to systematically assess the metabolic alterations in response to diarrhea-predominant IBS (IBS-D) and therapeutic effect of HPM.

Methods: Proton nuclear magnetic resonance spectroscopy ('H NMR)-based metabolomics approach was used to investigate fecal and serum metabolome of rat model of IBS-D with and without HPM treatment.

Results: The current results showed that IBS-induced metabolic alterations in fecal and serum sample include higher level of threonine and UDP-glucose together with lower levels of aspartate, ornithine, leucine, isoleucine, proline, 2-hydroxy butyrate, valine, lactate, ethanol, arginine, 2-oxoisovalerate and bile acids. These altered metabolites potentially involve in impaired gut secretory immune system and intestinal inflammation, malabsorption of nutrients, and disordered metabolism of bile acids. Notably, the HPM treatment was found able to normalize the Bristol stool forms scale scores, fecal water content, plasma endotoxin level, and a number of IBS-induced metabolic changes.
\end{abstract}

Conclusions: These findings may provide useful insight into the molecular basis of IBS and mechanism of the HPM intervention.

Keywords: Irritable bowel syndrome, Herb-partitioned moxibustion, Metabolomics, Nuclear magnetic resonance

*Correspondence: jingjing@xmu.edu.cn; xrchang1956@163.com

${ }^{\dagger}$ Xianwei Lin and Xia Liu contributed equally to this work

1 Department of Electronic Science, Fujian Provincial Key Laboratory

for Plasma and Magnetic Resonance, Xiamen University, Xiamen 361005,

China

${ }^{2}$ College of Acupuncture and Moxibustion, Hunan University of Chinese

Medicine, Changsha 410208, China

Full list of author information is available at the end of the article

\begin{abstract}
Background
Irritable bowel syndrome (IBS) is a chronic functional gastrointestinal disorder [1] characterized by stomachache, abdominal distension and altered bowl evacuation habit, along with abnormal fecal character [2]. The pathophysiology of IBS is multifactorial including gut motility, visceral hypersensitivity, mucosal micro-inflammation, increases in intestinal permeability, psychosocial factors, and brain-gut dysfunctions [3]. Based on predominant bowel habit and Rome III criteria [4], IBS can be classified into four subgroups including diarrhea-predominant IBS (IBS-D), constipation-predominant IBS (IBS-C),
\end{abstract}

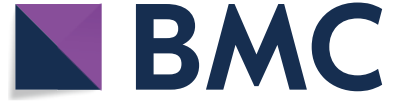

(c) The Author(s) 2019. This article is distributed under the terms of the Creative Commons Attribution 4.0 International License (http://creativecommons.org/licenses/by/4.0/), which permits unrestricted use, distribution, and reproduction in any medium, provided you give appropriate credit to the original author(s) and the source, provide a link to the Creative Commons license, and indicate if changes were made. The Creative Commons Public Domain Dedication waiver (http://creativecommons.org/ publicdomain/zero/1.0/) applies to the data made available in this article, unless otherwise stated. 
mixed diarrhea and constipation IBS (IBS-M) and unspecific IBS (IBS-U). Although IBS has a very low mortality rate, it poses a considerable medical, social and economic burden on individuals and societies [5].

The conventional medications for IBS provide relief for symptoms, however it may also lead to unwanted side effects. For example, a number of antidiarrhoeal and antispasmodics agents may lead to constipation; some serotonergic agents (e.g. 5-hydroxytryptamine ${ }_{3}$ and 5-hydroxytryptamine ${ }_{4}$ ) may result in severe constipation and ischaemic colitis; some laxative agents may induce increased bowel gas and abdominal distention [6]. Traditional Chinese medicine (TCM) also offers complementary and alternative therapies for IBS, and they were found to improve the IBS symptom with good feedback from patients [7]. Among the TCM treatments, herb-partitioned moxibustion (HPM) is a characteristic external therapy that has been repeatedly found to confer curative effect in ameliorating IBS symptoms [8]. However, the therapeutic mechanism of HPM on IBS is still unknown.

As a systematic approach, metabolomics has been used to study gastrointestinal disorders including IBS and metabolic response following interventions [9]. The ${ }^{1} \mathrm{H}$ high-resolution NMR spectroscopy has been widely used in metabolomics due to its nondestructive characteristics, high reproducibility and the requirement of minimal sample preparation [10]. In NMR-based metabolomics, peaks from a metabolite with given structural formula are determined by chemical shift (ppm) and intensities (proportional to the number of hydrogen atoms) in a ${ }^{1} \mathrm{H}$-NMR spectrum without the requirement of chromatographic separation and sample derivatization as in mass spectrometry-based metabolomics. The holistic strategy of NMR-based metabolomics is consistent with the systematic thinking of TCM, and thus may serve as a potential scientific platform to uncover the mechanism of evidence-based Chinese medicine including acupuncture and moxibustion [11-13]. In the present study, the blood serum and feces specimen obtained from a rat model of IBS-D and the metabolic impact of HPM treatment were investigated by a combination of high resolution NMR spectroscopy and multivariate statistical analysis. The aim of this study was to investigate the metabolic basis of HPM treatment in improving symptoms of IBS.

\section{Methods}

The Minimum Standards of Reporting Checklist (Additional file 1) contains details of the experimental design, and statistics, and resources used in this study.

\section{Ethical statement}

Animal care and experimental procedures used in the current study were approved by the Institutional Animal
Care and Use Committee of Hunan University of Chinese Medicine (Ethics No. SCXK 2011-0003). This study was conducted in accordance with local guidelines provided by National Institutes of Health for the Care and Use of Laboratory Animals and all efforts were made to minimize suffering of animals.

\section{Animal handling}

In the present study, forty male Sprague-Dawley rats (aged 4 weeks with body weight $180 \pm 10$ g) were housed individually in metabolism cages and provided with food and water ad libitum. The animal room was under controlled condition (temperature $22 \pm 1{ }^{\circ} \mathrm{C}$, humidity $40-60 \%$ and a 12 -h light-dark cycle). After acclimatization for 1 week, all rats were divided randomly into normal control rats $(\mathrm{NC}, n=10)$ and other rats $(n=32)$ which were used for IBS modelling.

After being fasted for $24 \mathrm{~h}$, all rats were deeply anaesthetized with a ether $(300 \mathrm{mg} / \mathrm{kg})$. The normal rats were injected with $0.9 \%$ saline solution $(5 \mathrm{mg} / \mathrm{kg})$ into the colon via PE90 tubing inserted to a depth of $8 \mathrm{~cm}$ from the anus. The IBS rat models were induced by colorectal administration of $4 \%$ acetic acid $(5 \mathrm{mg} / \mathrm{kg})$ in a similar manner to the normal rats followed by washing with $0.01 \mathrm{M}$ phosphate buffer saline $(5 \mathrm{mg} / \mathrm{kg})$ [14]. Seven days later, the IBS rats were treated with restraining stress for 2 weeks ( $1 \mathrm{~h}$ per day). These procedures are considered a reliable method for induction of IBS-D in rats [15]. Then, the rats with confirmed IBS-D were randomly divided into three groups, including untreated IBS rats (IBS group, $n=11$ ), or IBS rats that received herbpartitioned moxibustion at traditional acupoints (HPM group, $n=10$ ) or received pinaverium bromide treatment (PBT group, $n=11$ ) [16].

\section{Herb-partitioned moxibustion}

The herbal powder formula used in the HPM treatment was as follow: Baizhu (Rhizoma atractylodis) (500 g), Baishao (Radix paeoniaealba) (500 g), Chenpi (Pericarpium citrireticulatae) (500 g), and Fangfeng (Radix saposhnikoviae divaricatae) (500 g). The combination of these four herbs is used in the "Tongxieyaofang", a traditional Chinese medical prescription widely adopted in the clinical treatment of IBS-D [17]. Based on TCM understanding, the herbs collectively help to regulate liver function and rectify the spleen functions.

These traditional Chinese medicines were mixed, ground into a fine powder and stored in dry condition at room temperature $\left(25{ }^{\circ} \mathrm{C}\right)$. Then, $2.5 \mathrm{~g}$ of powder was mixed with $3 \mathrm{~g}$ of vinegar into herbal paste, and then shaped into herbal cake $(1 \mathrm{~cm}$ in diameter, $0.3 \mathrm{~cm}$ in height) using a herbal cake mold. Moxi cones (10 mg in weight, $0.5 \mathrm{~cm}$ in diameter, $1.5 \mathrm{~cm}$ in height) were 
hand-made by using refined mugwort floss (Huatuo, Suzhou, China).

Rats of the HPM group received the same fixation and hair located around the acupoints was cut off to expose the local skin before treatment. The acupoints of bilateral Ganyu (BL18), Piyu (BL20), Zusanli (ST36), Zhangmen (LR13) and Qimen (LA14) were selected for stimulation as these acupoints were found effective in relieving IBS symptoms in clinical practice [18]. According to the "Experimental Acupuncture and Moxibustion Science" combined with anthropomorphic analogy, Ganshu (BL18) acupoint is located below the ninth thoracic vertebra and $5 \mathrm{~mm}$ lateral to posterior midline. Pishu (BL20) is below the twelfth thoracic vertebra and $5 \mathrm{~mm}$ lateral to posterior midline. Zusanli (ST36) is located $5 \mathrm{~mm}$ below the fibular head and lateral to the anterior tubercle of the tibia. Zhangmen (LR13) is in front of the eleventh rib. Qimen (LA14) is located in the sixth intercostals space. The moxa cones were fixed to the herbal cake that was placed directly onto the acupoints. Five acupoints were stimulated (a 20-min session per day) one by one for 2 weeks. Burning injury was carefully avoided in the process by whisking way the burning ash in a timely manner.

For the PBT group, the solution of pinaverium bromide, $50 \mathrm{mg} /$ tablet (Abbott, USA), was prepared at the concentration of $13.5 \mathrm{mg} / \mathrm{kg}$ and was fed in dose of $10 \mathrm{~mL} / \mathrm{kg}$ by lavage once per day.

\section{Fecal water content and plasma endotoxin}

After the treatment, stool forms of the rats were observed and scored according to the Bristol Stool Form (BSF) scale [19]. Fecal water content (FWC) was also examined to estimate colonic motility as a validated index. Briefly, the stool was weighed after collection, and weighed again after drying in the oven. FWC was calculated as follow: water content $(\%)=100 \times($ wet weight - dry weight $) /$ wet weight. Besides, plasma samples were collected for determination of endotoxin by using a commercially available kit for tachypleus amebocyte lysate (TAL) technique (Zhanjiang A\&C Biological Ltd, Zhanjiang, China).

\section{Biological sample preparation}

After the 2-week treatment course, fecal samples from all rats were collected, snap-frozen in liquid nitrogen and stored at $-80^{\circ} \mathrm{C}$ until further metabolite extraction. The aqueous metabolites in fecal samples were extracted according to the protocol reported by Shao [20]. Thawed stool sample weighed $200 \mathrm{mg}$ was homogenized with $1.50 \mathrm{~mL}$ phosphate buffer solution (1.0 $\mathrm{M} \mathrm{K}_{2} \mathrm{HPO}_{4} /$ $\mathrm{NaH}_{2} \mathrm{PO}_{4}, \mathrm{pH} 7.4,99.9 \% \mathrm{D}_{2} \mathrm{O}$ ) containing $0.3 \mathrm{mM}$ TSP (3-(trimethylsilyl) propionic-2,2,3,3-d4 acid sodium salt) as an internal reference standard and vortexed for $60 \mathrm{~s}$. The mixed slurry was subjected to freeze-thaw ( 3 times) cycle by liquid nitrogen and followed by ultrasonication with a cycle of $20 \mathrm{~s}$-vortexing - $10 \mathrm{~s}$-waiting (20 times) in an ice bath. After $15 \mathrm{~min}$ of centrifugation $(10,000 \times \mathrm{g}$, $\left.4{ }^{\circ} \mathrm{C}\right), 600 \mu \mathrm{L}$ of the supernatant was transferred to centrifugal tube and remaining residue was further subjected to the above-mentioned procedure again. An equal volume of the second extracts from the same samples were mixed with the first extracts and then centrifuged at $10,000 \times g$ for $15 \mathrm{~min}$ at $4{ }^{\circ} \mathrm{C}$. A total of $550 \mu \mathrm{L}$ supernatant was transferred into $5 \mathrm{~mm}$ nuclear magnetic resonance (NMR) tube (Norell, ST50-7, USA) for NMR experiment. In the current study, we focused on the changes in body metabolism induced by moxibustion on the IBS-D model. The lipophilic metabolites in feces were not included in our analysis as they are mainly come from exogenous lipids intake of food and gut microbiome.

For blood samples, whole blood was obtained from abdominal aorta of the animals using a catheter and left to clot at room temperature $\left(22{ }^{\circ} \mathrm{C}\right)$ for $1 \mathrm{~h}$. For serum sample, a volume of $400 \mu \mathrm{L}$ was mixed with $200 \mu \mathrm{L}$ phosphate buffer solution $\left(90 \mathrm{mM} \mathrm{K}_{2} \mathrm{HPO}_{4} / \mathrm{NaH}_{2} \mathrm{PO}_{4}, \mathrm{pH}\right.$ 7.4, 99.9\% $\mathrm{D}_{2} \mathrm{O}$ ) without TSP. Then the mixture was transferred to centrifugal tube and centrifuged at $10,000 \times g$ for 15 min at $4{ }^{\circ} \mathrm{C}$. A total of $550 \mu \mathrm{L}$ supernatant was transferred into $5 \mathrm{~mm}$ NMR tube for NMR experiment.

\section{NMR experiments}

${ }^{1} \mathrm{H}$ NMR spectra of the fecal and serum samples were acquired at $296 \mathrm{~K}$ using a Bruker $600 \mathrm{MHz}$ NMR spectrometer (Bruker Biospin, Germany) equipped with a cryogenic inverse detection probe. The NMR spectra of fecal samples were recorded using the NOESYPR1D pulse sequence [21]. For the serum sample, an additional Carr-Purcell-Meiboom-Gill (CPMG) spin-echo pulse train [22] was incorporated into the NOESYPR sequence with a relaxation time $(2 \mathrm{nt})$ of $100 \mathrm{~ms}$ and an echo time

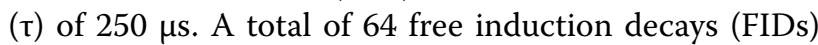
were collected for each NMR spectrum into $64 \mathrm{~K}$ data points over a spectral width of $12,000 \mathrm{~Hz}$ with a relaxation delay of $6.5 \mu \mathrm{s}$ and a mixing time of $120 \mathrm{~ms}$.

\section{Data preprocessing and multivariate statistical analysis}

The acquired ${ }^{1} \mathrm{H}$ NMR spectra were phase- and baseline-corrected using the MestReNova v 9.0.1 software (Mestrelab Research S.L.). Each spectrum was referenced to TSP at $\delta 0.00$ and peaks were aligned manually to overcome peak-shift problem [23]. With the aid of 2D NMR spectroscopy including ${ }^{1} \mathrm{H}-{ }^{1} \mathrm{H}$ correlation spectroscopy (COSY) [24], ${ }^{1} \mathrm{H} \mathrm{J}$-Resolved and ${ }^{1} \mathrm{H}-{ }^{13} \mathrm{C}$ heteronuclear single quantum correlation (HSQC) together with previous literatures [25], the major peaks in spectra were identified and assigned to specific metabolites. 
Prior to statistical analysis, these spectral data were normalized by using the probabilistic quotient normalization (PQN) method [26] to compensate for differences in overall concentrations of samples. In contrast to traditional integral normalization that takes the total integral of all signals as a function of dilution, the PQN method calculates a most probable quotient between signals of the corresponding spectrum and of a reference spectrum (normally the median spectrum of control samples) and takes it as normalization factor to account for dilution variation among samples. The spectral deconvolution method, also known as the target profiling [27], was performed to obtain the concentration of known metabolite signals in the spectra. Each spectrum was then matched in accordance with the respective signals of metabolites in standard compound library of the Chenomx NMR suite (ver 8.2, Chenomx Inc.).

Next, the quantified NMR data were exported as Microsoft Excel files and imported into the SIMCA software (version 14.1, U metrics AB, Umea, Sweden) for multivariate data analysis. The partial least squares discriminant analysis (PLS-DA) were used to examine metabolic differences due to different interventions at the molecular level. Validation of these models were performed with a seven-fold cross-validation and permutation test (200 permutations) [28]. In addition, the fold-change and Student's $t$ test with a Bonferroni correction were used for univariate analysis. Parameters from both multivariate and univariate analysis were embedded into one single volcano plot as described in previous studies [29].

\section{Pathway analysis}

The metabolite set enrichment analysis and pathway analysis were performed using the MetaboAnalyst v4.0 (http://www.metaboanalyst.ca) [30]. In the analysis, the Rattus norvegicus (rat) pathway library was chosen, and the Fisher's exact test was selected for the over-representation analysis, and relative betweenness centrality was chosen for topology analysis.

\section{Results}

\section{Stool forms evaluation and plasma endotoxin assays}

Microscopic examinations were conducted to study the effects of HPM or PBT treatment on morphology of intestines. As shown in Additional file 2: Figure S1, little pathological changes were observed in each group by the intestinal HE staining. The result is consistent with a previous study on intestinal permeability of IBS-D rats by administration of acetic acid [31]. The current findings indicated that the presence of diarrhea symptoms and metabolic changes are more sensitive to gastrointestinal disorder, and they occurred earlier than pathological changes in intestines.

Following disease modeling, the mean BSF scale scores and FWC (Fig. 1) of the IBS group were found significantly higher $(p<0.05)$ than the control NC group. This is consistent with the observation of deformed sloppy stool excreted by the IBS rats. The data indicated that the modelling of IBS-D was established successfully [32]. After treatments with either HPM or PBT, the animals showed significant decreased BSF scale scores $(p<0.05)$ and FWC $(p<0.05)$ with a firmer stool form as compared to the IBS group. By comparing the HPM and NC rats, there are no

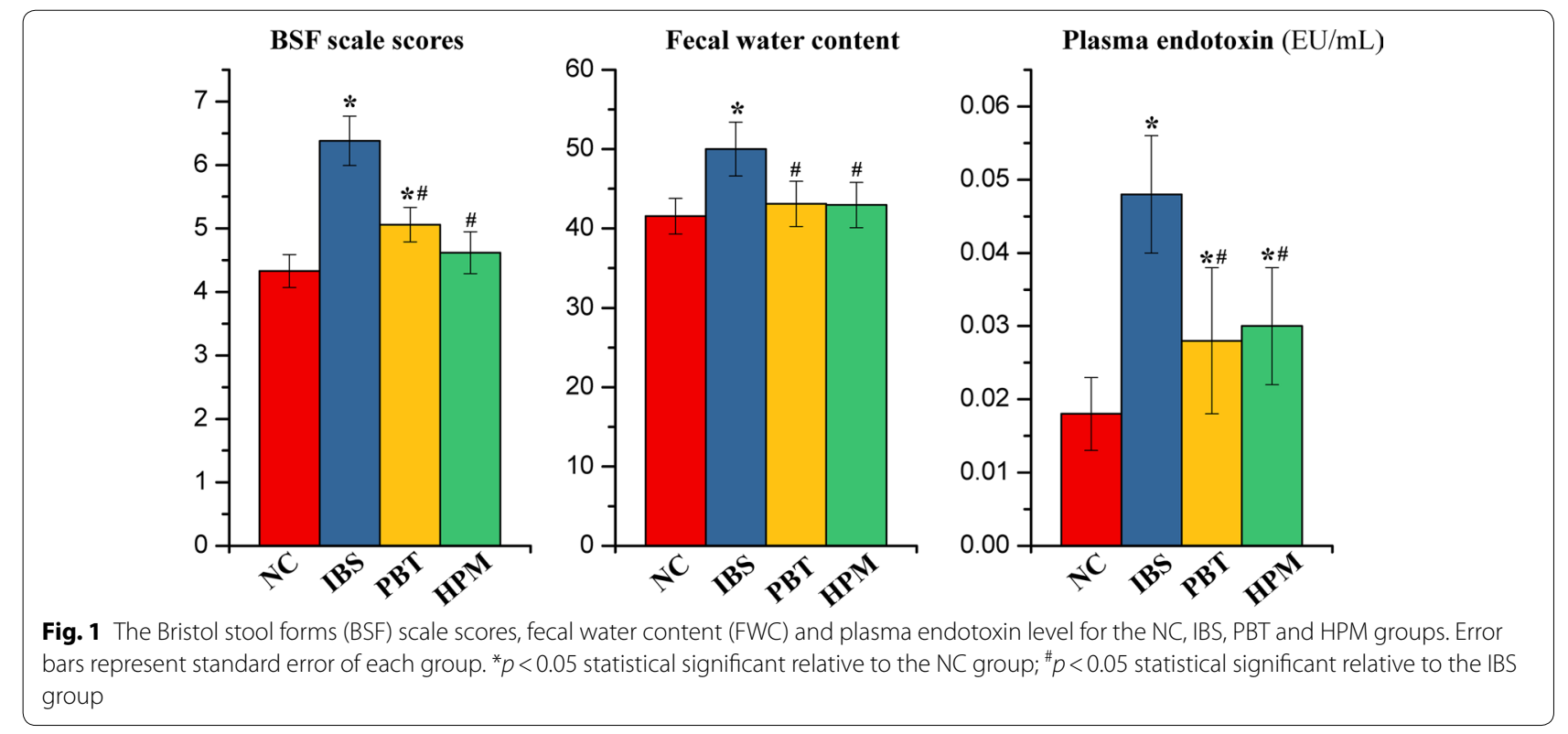


significant difference in BSF and FWC, suggesting HPM treatment is efficient in alleviating diarrhea symptoms. In contrast, PBT treatment reversed the FWC to normal but the BSF of the PBT group remained higher $(p<0.05)$ than the normal controls.

Endotoxins, also known as lipopolysaccharides (LPS), is an indicator for micro-inflammation of the intestinal mucosa that plays a critical role in the pathogenesis of IBS [33]. Plasma endotoxin levels in IBS group was found markedly increased by $166 \%(p<0.05)$ after IBS modelling (Fig. 1). This finding indicated that endotoxins entered the blood circulation due to an altered permeability of the intestinal barrier in the IBS animals. Both treatments reduced the endotoxin level induced by IBS $(p<0.05)$ although the values are still higher than the NC group $(p<0.05)$. The result suggested partial reversal of intestinal inflammation due to either HPM or PBT treatment.

\section{${ }^{1} \mathrm{H}$ NMR profiling of feces and serum from rats}

Typical ${ }^{1} \mathrm{H}$ NMR spectra of fecal extracts and serum are shown in Additional file 2: Figures S2 and S3. Both unsupervised (PCA) and supervised pattern recognition
(PLS-DA) were applied on the NMR data of feces and serum data to observe the clustering tendency of samples due to different interventions (Fig. 2). Particularly, one fecal sample from the $\mathrm{NC}$ group was lost during handling and hemolysis was observed in three serum samples (one each from the IBS, HPM and PBT group), therefore they were excluded from the following analysis. For both feces and serum datasets, legible group separation with slightly overlapping can be seen in PCA score plots for the NC and IBS groups, indicating significant metabolic variations induced by colorectal administration of acetic acid and restraint stress. Points of fecal samples from rats with HPM treatment are located close to the controls whereas points from PBT groups are totally mixed with the IBS ones. Thus IBS-induced metabolic perturbations are suggested to be reversed partially towards healthy state following HPM treatment but little affected by PBT treatment. Scattered points of the PBT group in serum score plot show individual variations of metabolic patterns responding to drug intervention. However, the HPM group shows inter-group separation from the NC and IBS groups and specific intra-group gathering with one point deviating from $95 \%$ confidence interval. With
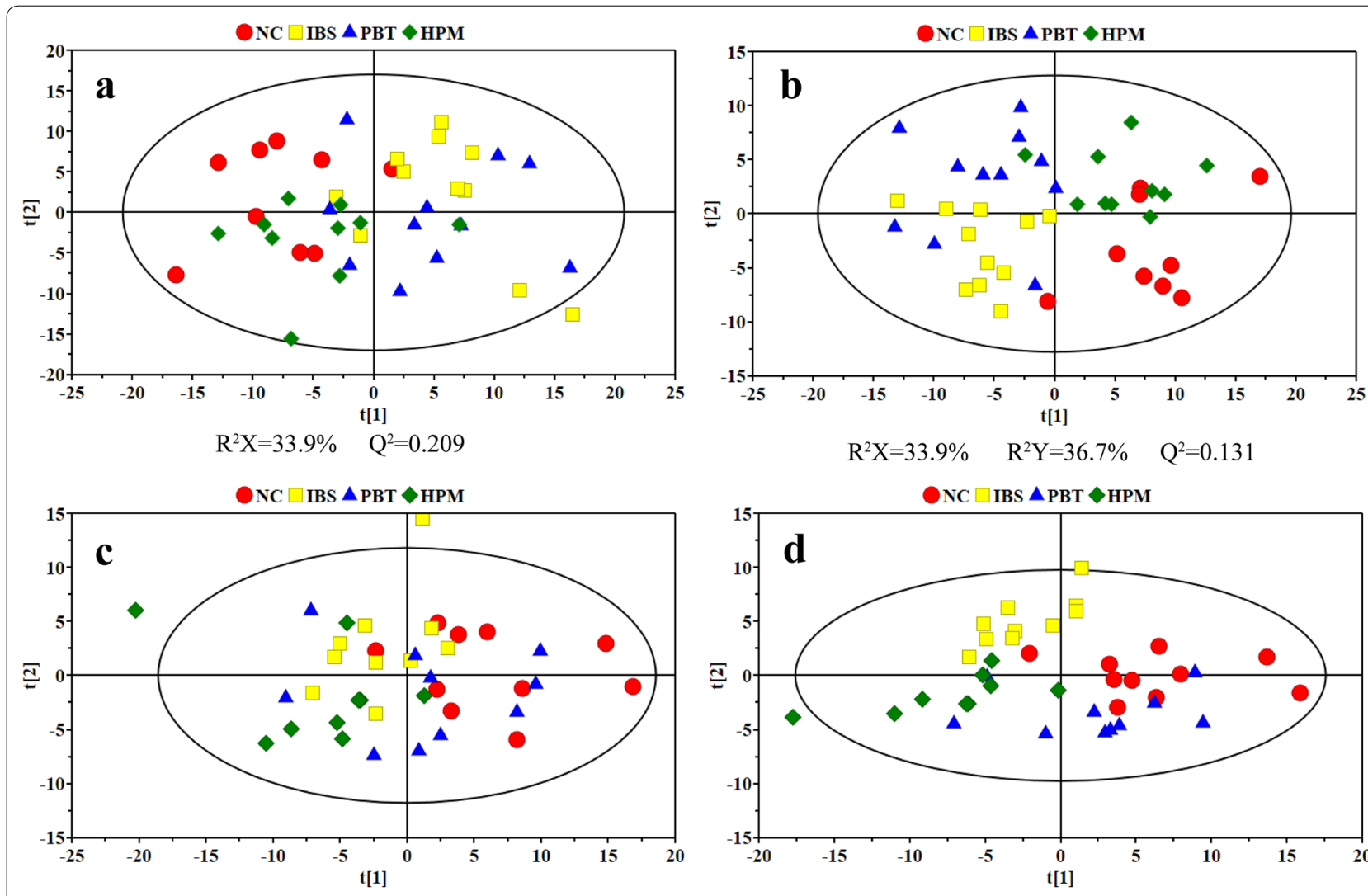

$\mathrm{R}^{2} \mathrm{X}=37.7 \% \quad \mathrm{Q}^{2}=0.233$

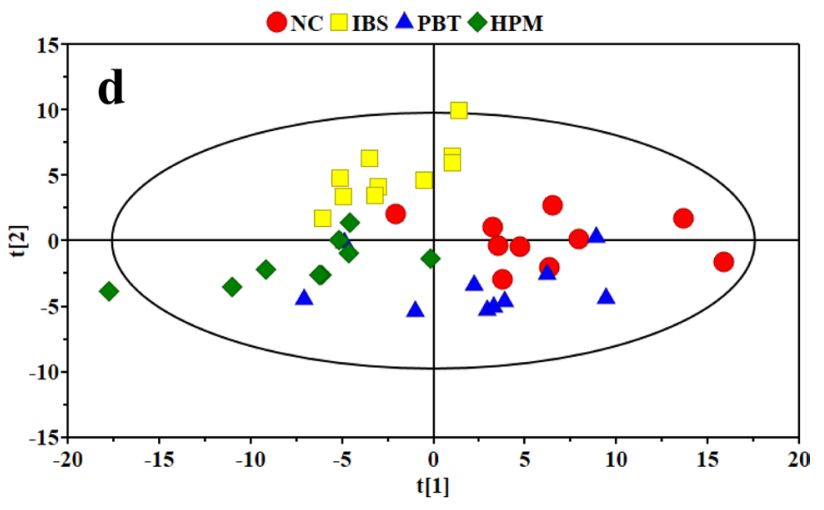

$\mathrm{R}^{2} \mathrm{X}=34.7 \% \quad \mathrm{R}^{2} \mathrm{Y}=43.4 \% \quad \mathrm{Q}^{2}=0.269$

Fig. 2 Scores plots of PCA (left column) and PLS-DA (right column) models derived from NMR datasets of feces (a, b) and serum (c, d) 
combining the supervised class information (Y variable), visible separation of these four groups were improved in PLS-DA score plots (Fig. 2b, d).

\section{Identification of metabolic alterations in feces and serum}

To further investigate the metabolic impact of HPM treatment on the IBS rats, PLS-DA models were conducted on feces (Fig. 3) and serum (Fig. 4) data acquired from healthy controls, IBS rats, and IBS rats with the HPM treatment. An eightfold cross validation and random permutation tests (200 times) were carried out on the PLS-DA models to validate the robustness of the resulting PLS-DA models.

PLS-DA of fecal dataset showed a clear group separation between the NC and the IBS groups (Fig. 3a). An enhanced volcano plot, which integrates model parameters of multivariate analysis (correlation coefficient and variable importance projection) with statistical parameters of univariate (fold change and $p$-value), was used to identify candidate metabolites that contributed to the inter-group separation. Following the IBS modelling, metabolic alterations in fecal metabolome include increased concentrations of threonine and UDP-glucose together with decreased concentrations of leucine, isoleucine, proline, 2-hydroxybutyrate, valine, lactate, ethanol, arginine, 2-oxoisovalerate and bile acids (Fig. 3c).

The PLS-DA score plot of fecal samples from the NC and HPM group is shown Fig. 3d. Although separation between these two groups can be observed along the $t$ [1] dimension, this PLS-DA model has failed in cross validation (Fig. 3e), suggesting high similarity in metabolome for both groups. For this PLS-DA model, volcano plot was not constructed as differential metabolites cannot be identified based on this unreliable discriminant model. The current results supported that HPM treatment may re-normalize the IBS-induced metabolic changes in rats.

Analysis of the blood serum dataset using PLS-DA showed good separation between the NC and the IBS groups (Fig. 4a), and between the NC and the HPM groups (Fig. 4d). These two models were found to be robust by a sevenfold cross validation and permutation test (200 times) (Fig. 4b, e). For serum metabolome, metabolic changes in the IBS group were highlighted with significant decrease of aspartate and ornithine. By contrast, the HPM group shows significant increased levels of lactate, alanine, 1-methylhistidine, valine and leucine
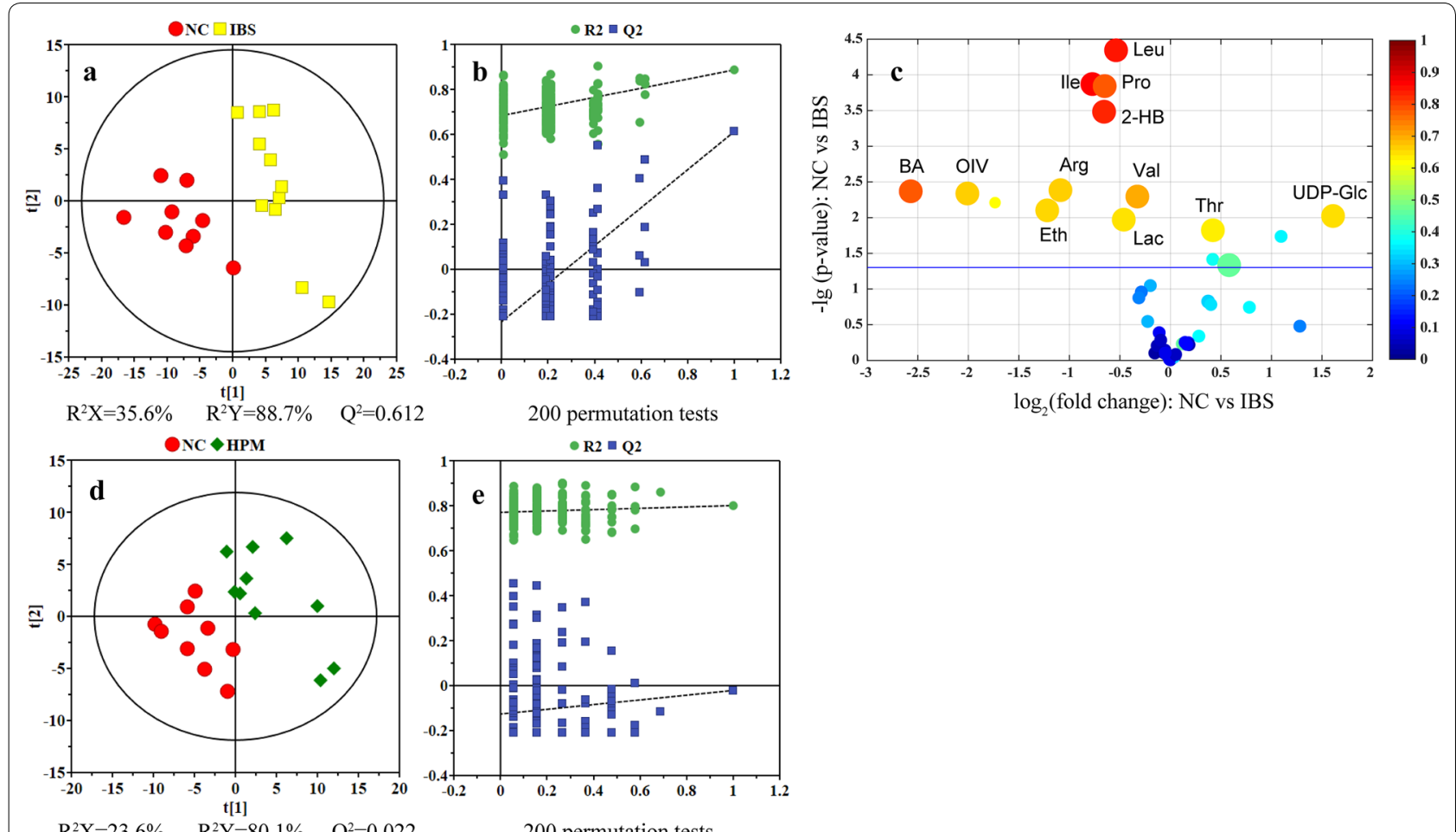

$\log _{2}$ (fold change): NC vs IBS

Fig. 3 PLS-DA scores plots (left panels), their corresponding coefficient loading plots (middle panels) and volcano plots (right panels) derived from fecal NMR data. Variable importance projection (VIP) together with correlation coefficient of each model is introduced with being represented by circle size and color. VIP values are categorized into two segments: top $20 \%$ and rest $80 \%$ represented by a circle of larger or small size. Leu, leucine; Ile, isoleucine; Pro, propionate; 2-HB, 2-hydroxybutyrate; BA, bile acids; OIV, 2-oxoisovalerate; Arg, arginine; Val, valine; Eth, ethanol; Lac, lactate; Thr, threonine; UDP-Glc, uridine diphosphate glucose 

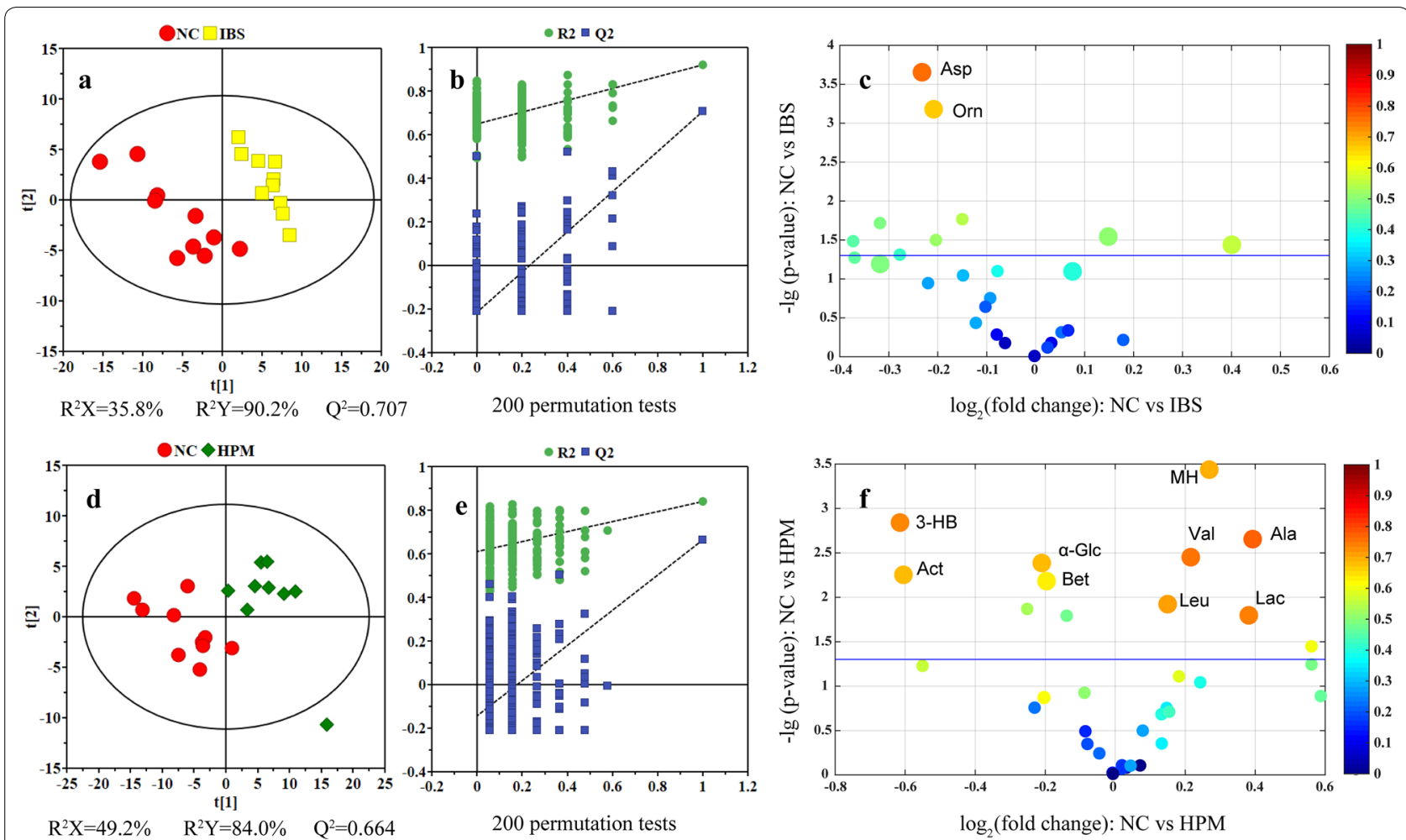

Fig. 4 PLS-DA score plots (left panels), their corresponding coefficient loading plots (middle panels) and volcano plots (right panels) derived from serum NMR data. Leu, leucine; Val, valine; Lac, lactate; Asp, aspartate; Orn, ornithine; 3-HB, 3-hydroxybutyrate; MH, 1-methylhistidine; Act, acetone; a-Glc; a-glucose; Ala, alanine; Bet, betaine

together with decreased levels of betaine, $\alpha$-glucose, 3-hydroxybutyrate and acetone.

\section{Discussion}

The current study used high-resolution NMR spectroscopy with multivariate statistical analysis to investigate metabolic perturbation in fecal extracts and serum samples from IBS rats with/without HPM treatment. In general, we observed relatively mild IBS-induced metabolic perturbation in the serum metabolome. In contrast, biochemical components in feces correlated directly to intestinal morphology and intestinal flora so that fecal metabolome is more sensitive to intestinal biological changes in current IBS animal model. Based on differential metabolites identified using volcano plots, an enrichment analysis of metabolic pathway was performed by the online MetaboAnalyst (Metabolomics Pathway Analysis) toolbox [30]. As shown in Fig. 5a, arginine and proline metabolism, urea cycle as well as valine, leucine and isoleucine degradation are considered to be the key three pathways changed by IBS modelling. Then, these differential metabolites are mapped onto the three metabolic pathways (Fig. 5b). Systematic metabolic alterations related to multiple biological functions were found to be changed by chemical-induced intestinal inflammation, which could be partially regulated by HPM treatment. Histograms of characteristic metabolites corresponding to the concentrations in each group were embedded into the plot to show the trends of changes.

Fecal concentration of threonine was found higher in the IBS group than the controls $(p<0.05)$. This metabolic change can be reversed by either HPM or PBT treatment $(p<0.05)$. Threonine is an essential amino acid in intestine canal that involves in the production of mucin [34] and immunoglobulin A (IgA) [35] to maintain the intestinal homeostasis. IBS-induced inflammation may facilitate the threonine utilization for synthesis of intestinal mucins and IgA [36]. Therefore, the increased threonine in feces may be due to impaired gut secretory immune system with limited luminal threonine availability upon exposure to inflammatory stimuli. This metabolic perturbation of intestinal homeostasis can be normalized by using either HPM or PBT treatment.

Aspartate, proline, ornithine and arginine are generally considered as the amino acids of arginine family. The amino acids are inter-convertible via inter-organ metabolism in mammals [37]. These four metabolites were observed to be reduced in fecal samples upon 


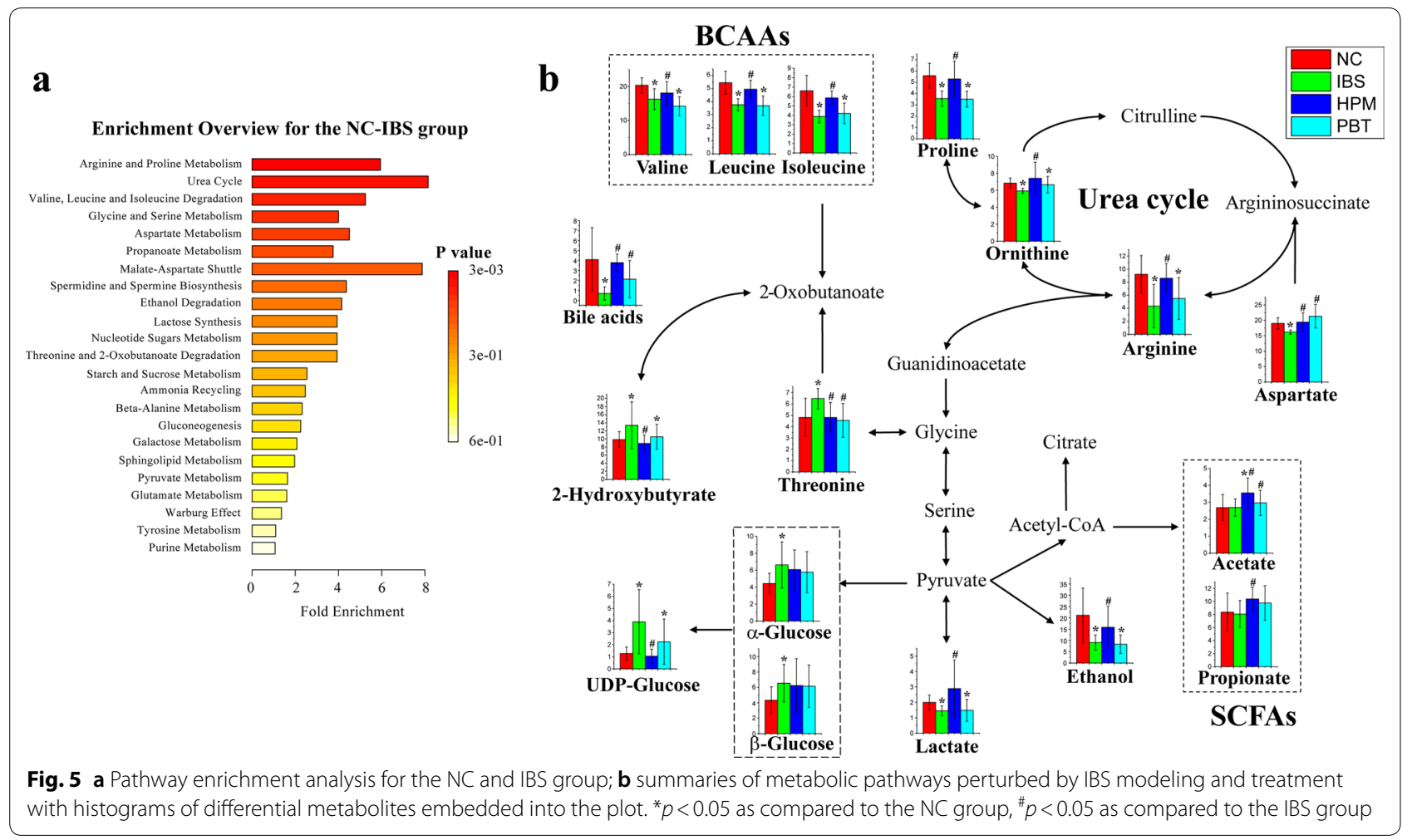

IBS modelling, and can be returned to normal after 2-week HPM treatment. Notably, aspartate is one of the major sources of ATP in mammalian enterocytes via mitochondrial oxidation [38] and play a critical role in inhibiting NODs/NF- $\mathrm{kB}$ and p38 signaling pathways to improve the intestinal integrity under an inflammatory condition [39]. On the other hand, arginine involves in ornithine cycle to promote urea formation, and this was found to exert vital function on intestinal inflammation via immune response and oxidative system [40]. Based on the previous reports [41], reductions in these arginine-group amino acids following IBS-modelling can be considered as an indicator of immunological stress due to intestinal injury of acetate administration.

Valine, leucine and isoleucine, known as branchedchain amino acids (BCAAs), are beneficial to maintain intestinal barrier integrity and protect intestinal mucosal permeability in absorption of nutrients [42]. Decreased concentrations of BCAAs in feces of the IBS rats may be accounted for the malabsorption of nutrients due to epithelium inflammation and injury by particular bowel pathology [43]. HPM treatment was found to significantly increase BCAAs but PBT treatment only led to minor changes compared to IBS group, supporting the holistic metabolic regulation of HPM in relieving intestinal injury and improving intestinal function.
Metabolism of bile acids has been reported to be close interacted with gut microbiome and intestinal inflammation [44]. The fecal bile acids pool composition is determined by two bacterial metabolic reactions [45]: firstly, the deconjugation of the amino acid and secondly, the transformation of primary bile acids into secondary bile acids. Dior et al. [46] reported a decreased ability for bile acids deconjugation (first step) in IBS feces compared to healthy subjects using an in vitro test. Thus, the decreased bile acids in feces of IBS rats is speculated to be correlated with the decrease in bile acid biotransformation and passively reabsorbed in the ileum. The dysregulated metabolism of bile acids was found improved by either HPM or PBT treatment.

Energy-related metabolites in the human intestine are crucial to gut microbiome and intestinal cells. Glucose metabolism in hosts can regulate $\mathrm{T}$ cell activation [47] and UDP-glucose can stimulate IL-8 production via the P2RY14 receptor [48]. Increased glucose and UDP-glucose levels observed in fecal samples of the IBS animals may be resulted from the disturbance in the host-microbiome system, where HPM treatment was found effective in regulating UDG-glucose level to normal.

Short-chain fatty acid (SCFAs) including acetate and propionate are readily absorbed and used as an energy source by colonocytes [49]. SCFAs provide an important defensive capacity against colorectal carcinogenesis by 
reducing epithelial inflammation and triggering cancer cell apoptosis via p21 activity [50]. In the current study, the concentrations of acetate and propionate in feces were comparable between the IBS group and healthy controls. However, HPM treatment led to increased levels of these two SCFAs, compared with the IBS group. This result indicated that HPM may confer protective mechanism on intestinal microbiota and host tissues upon inflammation and tumorigenesis.

\section{Conclusions}

In conclusion, ${ }^{1} \mathrm{H}$ NMR spectroscopy-based metabolomics was used to characterize the metabolome variations in blood serum and fecal samples following IBS modelling and HPM/PBT treatment. The altered metabolic pathways in IBS rats revealed the disruption of intestinal homeostasis, dysregulated immune response, malabsorption of nutrients and perturbed bile acids metabolism, which may be associated with IBS. Traditional moxibustion treatment with herbal medicine showed beneficial effects by re-normalizing IBS-induced metabolic changes.

\section{Additional files}

Additional file 1. The Minimum Standards of Reporting Checklist.

Additional file 2. NMR spectra of fecal extracts and serum.

\section{Abbreviations}

IBS: irritable bowel syndrome; IBS-D: diarrhea-predominant irritable bowel syndrome; IBS-C: constipation-predominant irritable bowel syndrome; IBS-M: mixed diarrhea and constipation irritable bowel syndrome; IBS-U: unspecific irritable bowel syndrome; TCM: traditional Chinese medicine; HPM: herbpartitioned moxibustion; NC: normal control; BSF: Bristol Stool Form; FWC: fecal water content; TAL: tachypleusamebocyte lysate; TSP: 3-(trimethylsilyl) propionic-2,2,3,3-d4 acid sodium salt; NMR: nuclear magnetic resonance; CPMG: Carr-Purcell-Meiboom-Gill; FID: free induction decay; COSY: correlation spectroscopy; HSQC: heteronuclear single quantum correlation; PLS-DA: partial least squares discriminant analysis; LPS: lipopolysaccharides; PCA: principal component analysis; IgA: immunoglobulin A; BCAAs: branched-chain amino acids; SCFAs: short-chain fatty acids.

\section{Authors' contributions}

$J J X$ and XRC conceived and designed the study; XWL and XL wrote the main manuscript text; JNC, TL and QL performed the animal experiments; HZ, GPS and JYD analyzed the data and prepared the figures; KKC revised the manuscript. All authors read and approved the final manuscript.

\section{Acknowledgements}

Not applicable.

\section{Competing interests}

The authors declare that they have no competing interests.

\section{Availability of data and materials}

The datasets used during the current study are available from the corresponding author on reasonable request.

\section{Consent for publication}

All authors have provided consent for publication in the Journal of Chinese Medicine.

\section{Ethics approval and consent to participate}

Animal care and experimental procedures used in the current study were approved by the Institutional Animal Care and Use Committee of Hunan University of Chinese Medicine (Ethics No. SCXK 2011-0003).

\section{Funding}

This study was supported by National Natural Science Foundation of China (Nos. 81871445, 81801788, 81603705, 81674084, 81874509), the National Key Basic Research Development Program of China (No. 2015CB554502). KKC is supported by Flagship Research University Grant from Universiti Teknologi Malaysia (R.J130000.2446.03G58)

\section{Author details}

${ }^{1}$ Department of Electronic Science, Fujian Provincial Key Laboratory for Plasma and Magnetic Resonance, Xiamen University, Xiamen 361005, China. ${ }^{2}$ College of Acupuncture and Moxibustion, Hunan University of Chinese Medicine, Changsha 410208, China. ${ }^{3}$ Innovation Centre in Agritechnology, Universiti Teknologi Malaysia, 84600 Muar, Johor, Malaysia.

Received: 30 January 2019 Accepted: 29 April 2019

Published online: 08 May 2019

\section{References}

1. Gwee K. Irritable bowel syndrome in developing countries-a disorder of civilization or colonization? Neurogastroent Motil. 2010;17(3):317-24.

2. Keszthelyi D, Troost FJ, Masclee AA. Irritable bowel syndrome: methods, mechanisms, and pathophysiology. Methods to assess visceral hypersensitivity in irritable bowel syndrome. Am J Physiol-Gastr L. 2012;303(2):G141-54

3. Canavan C, West J, Card T. The epidemiology of irritable bowel syndrome. Clin Epidemiol. 2014;6:71-80.

4. Drossman DA. Functional gastrointestinal disorders: history, pathophysiology, clinical features, and Rome IV. Gastroenterology. 2016;150(6):1262-79.

5. Nellesen D, Yee K, Chawla A, Lewis BE, Carson RT. A systematic review of the economic and humanistic burden of illness in irritable bowel syndrome and chronic constipation. J Manage Care Pharm. 2013;19(9):755-64.

6. Heading R, Bardhan K, Hollerbach S, Lanas A, Fisher G. Systematic review: the safety and tolerability of pharmacological agents for treatment of irritable bowel syndrome - a European perspective. Aliment Pharm Ther. 2006;24(2):207-36.

7. Bi Z, Zheng Y, Yuan J, Bian Z. The efficacy and potential mechanisms of chinese herbal medicine on irritable bowel syndrome. Curr Pharm Design. 2017;23(34):5163-72.

8. Ma Y-X, Liu X, Liu C-Z, Wang L-P, Guo G, Du D-Q, et al. Randomized clinical trial: the clinical effects of herb-partitioned moxibustion in patients with diarrhoea-predominant irritable bowel syndrome. Evid-Based Compl Alt. 2013. https://doi.org/10.1155/2013/605460.

9. Noorbakhsh $\mathrm{H}$, Yavarmanesh M, Mortazavi SA, Adibi P, Moazzami AA. Metabolomics analysis revealed metabolic changes in patients with diarrhea-predominant irritable bowel syndrome and metabolic responses to a synbiotic yogurt intervention. Eur J Nutr. 2018. https://doi.org/10.1007/ s00394-018-1855-2.

10. Markley JL, Bruschweiler R, Edison AS, Eghbalnia HR, Powers R, Raftery $D$, et al. The future of NMR-based metabolomics. Curr Opin Biotechnol. 2017:43:34-40

11. Zhang AH, Sun H, Wang ZG, Sun WJ, Wang P, Wang XJ. Metabolomics: towards understanding traditional chinese medicine. Planta Med. 2010;76(17):2026-35.

12. He QD, Huang YP, Zhu LB, Shen JC, Lian LY, Zhang Y, et al. Difference of liver and kidney metabolic profiling in chronic atrophic gastritis rats between acupuncture and moxibustion treatment. Evid 
Based Complement Alternat Med. 2018;2018:6030929. https://doi. org/10.1155/2018/6030929.

13. Liu CC, Chen JL, Chang XR, He QD, Shen JC, Lian LY, et al. Comparative metabolomics study on therapeutic mechanism of electro-acupuncture and moxibustion on rats with chronic atrophic gastritis (CAG). Sci Rep. 2017;7:1-11.

14. Chen JY, Chen Z, Ma L, Liang QW, Jia W, Pan ZZ, et al. Development of determination of four analytes of Zhi-Shao-San decoction using LC-MS/MS and its application to comparative pharmacokinetics in normal and irritable bowel syndrome rat plasma. Biomed Chromatogr. 2014;28(10):1384-92.

15. Shamshiri H, Paragomi P, Paydar MJ, Moezi L, Bahadori M, Behfar B, et al. Antinociceptive effect of chronic lithium on visceral hypersensitivity in a rat model of diarrhea-predominant irritable bowel syndrome: the role of nitric oxide pathway. J Gastroen Hepatol. 2009;24(4):672-80.

16. Zheng L, Lai YL, Lu WM, Li BW, Fan H, Yan ZX, et al. Pinaverium reduces symptoms of irritable bowel syndrome in a multicenter, randomized, controlled trial. Clin Gastroenterol H. 2015;13(7):1285-92.

17. Ma X, Wang X, Kang N, Chen T, Ji H, Lv L, et al. The effect of TongXie-Yao-Fang on intestinal mucosal mast cells in post infectious irritable bowel syndrome rats. Evid-Based Compl Alt. 2017. https://doi. org/10.1155/2017/9086034.

18. Bao CH, Zhang JZ, Liu JM, Liu HR, Wu LY, Shi Y, et al. Moxibustion treatment for diarrhea-predominant irritable bowel syndrome: study protocol for a randomized controlled trial. Bmc Complem Altern M. 2016;16:408.

19. Lewis SJ, Heaton KW. Stool form scale as a useful guide to intestinal transit time. Scand J Gastroentero. 1997;32(9):920-4.

20. Shao TJ, He ZX, Xie ZJ, Li HC, Wang MJ, Wen CP. Characterization of ankylosing spondylitis and rheumatoid arthritis using H-1 NMR-based metabolomics of human fecal extracts. Metabolomics. 2016;12(4):70.

21. Lamichhane S, Sundekilde UK, Blaedel T, Dalsgaard TK, Larsen LH, Dragsted LO, et al. Optimizing sampling strategies for NMR-based metabolomics of human feces: pooled vs. unpooled analyses. Anal Methods. 2017;9(30):4476-80.

22. Dona AC, Jimenez B, Schafer H, Humpfer E, Spraul M, Lewis MR, et al. Precision high-throughput proton NMR spectroscopy of human urine, serum, and plasma for large-scale metabolic phenotyping. Anal Chem. 2014;86(19):9887-94.

23. Veselkov KA, Lindon JC, Ebbels TMD, Crockford D, Volynkin W, Holmes $\mathrm{E}$, et al. Recursive segment-wise peak alignment of biological $\mathrm{H}-1$ NMR spectra for improved metabolic biomarker recovery. Anal Chem. 2009;81(1):56-66.

24. Aguilar JA, Belda R, Gaunt BR, Kenwright AM, Kuprov I. Separating the coherence transfer from chemical shift evolution in high-resolution pure shift COSY NMR. Magn Reson Chem. 2018;56(10):969-75.

25. Puig-Castellvi F, Perez Y, Pina B, Tauler R, Alfonso I. Comparative analysis of H-1 NMR and H-1-C-13 HSQC NMR metabolomics to understand the effects of medium composition in yeast growth. Anal Chem. 2018;90(21):12422-30.

26. Dieterle F, Ross A, Schlotterbeck G, Senn H. Probabilistic quotient normalization as robust method to account for dilution of complex biological mixtures. Application in H-1 NMR metabonomics. Anal Chem. 2006;78(13):4281-90.

27. Barding GA, Salditos R, Larive CK. Quantitative NMR for bioanalysis and metabolomics. Anal Bioanal Chem. 2012;404(4):1165-79.

28. Nakanishi-Ohno Y, Hukushima K. Data-driven diagnosis for compressed sensing with cross validation. Phys Rev E. 2018;98(5):052120.

29. Xu JJ, Zheng XJ, Cheng KK, Chang XR, Shen GP, Liu M, et al. NMR-based metabolomics reveals alterations of electro-acupuncture stimulations on chronic atrophic gastritis rats. Sci Rep. 2017;7:45580.

30. Chong J, Soufan O, Li C, Caraus I, Li SZ, Bourque G, et al. MetaboAnalyst 4.0: towards more transparent and integrative metabolomics analysis. Nucleic Acids Res. 2018;46(W1):W486-94.

31. Hou QK, Huang YQ, Zhu SL, Li PW, Chen XL, Hou ZK, et al. MiR-144 increases intestinal permeability in IBS-D rats by targeting OCLN and ZO1. Cell Physiol Biochem. 2017;44(6):2256-68.
32. Lin MJ, Yu BP. Role of high-affinity choline transporter 1 in colonic hypermotility in a rat model of irritable bowel syndrome. J Neurogastroenterol. 2018;24(4):643-55.

33. Ohman L, Tornblom H, Simren M. Crosstalk at the mucosal border: importance of the gut microenvironment in IBS. Nat Rev Gastro Hepat. 2015;12(1):36-49.

34. Law GK, Bertolo RF, Adjiri-Awere A, Pencharz PB, Ball RO. Adequate oral threonine is critical for mucin production and gut function in neonatal piglets. Am J Physiol-Gastr L. 2007;292(5):G1293-301.

35. Azzam MMM, Zou XT, Dong XY, Xie P. Effect of supplemental L-threonine on mucin 2 gene expression and intestine mucosal immune and digestive enzymes activities of laying hens in environments with high temperature and humidity. Poultry Sci. 2011;90(10):2251-6.

36. Faure M, Chone F, Mettraux C, Godin JP, Bechereau F, Vuichoud J, et al Threonine utilization for synthesis of acute phase proteins, intestinal proteins, and mucins is increased during sepsis in rats. J Nutr. 2007;137(7):1802-7.

37. Wu GY. Functional amino acids in nutrition and health. Amino Acids. 2013;45(3):407-11.

38. Wu GY, Wu ZL, Dai ZL, Yang Y, Wang WW, Liu C, et al. Dietary requirements of "nutritionally non-essential amino acids" by animals and humans. Amino Acids. 2013;44(4):1107-13.

39. Wang HB, Liu YL, Shi HF, Wang XY, Zhu HL, Pi DG, et al. Aspartate attenuates intestinal injury and inhibits TLR4 and NODs/NF-kappa $\mathrm{B}$ and p38 signaling in weaned pigs after LPS challenge. Eur J Nutr. 2017;56(4):1433-43.

40. Ren WK, Chen S, Yin J, Duan JL, Li TJ, Liu G, et al. Dietary arginine supplementation of mice alters the microbial population and activates intestinal innate immunity. J Nutr. 2014;144(6):988-95.

41. Tan JZ, Liu SS, Guo YM, Applegate TJ, Eicher SD. Dietary L-arginine supplementation attenuates lipopolysaccharide-induced inflammatory response in broiler chickens. Brit J Nutr. 2014;111 (8):1394-404.

42. Ren M, Zhang SH, Zeng XF, Liu H, Qiao SY. Branched-chain amino acids are beneficial to maintain growth performance and intestinal immunerelated function in weaned piglets fed protein restricted diet. Asian Austral J Anim. 2015;28(12):1742-50.

43. He F, Wu CL, Li P, Li NZ, Zhang D, Zhu QQ, et al. Functions and signaling pathways of amino acids in intestinal inflammation. Biomed Res Int. 2018. https://doi.org/10.1155/2018/9171905.

44. Pavlidis P, Powell N, Vincent RP, Ehrlich D, Bjarnason I, Hayee B. Systematic review: bile acids and intestinal inflammation-luminal aggressors or regulators of mucosal defence? Aliment Pharm Ther. 2015;42(7):802-17.

45. Ridlon JM, Kang DJ, Hylemon PB. Bile salt biotransformations by human intestinal bacteria. J Lipid Res. 2006;47(2):241-59.

46. Dior M, Delagreverie H, Duboc H, Jouet P, Coffin B, Brot L, et al. Interplay between bile acid metabolism and microbiota in irritable bowel syndrome. Neurogastroent Motil. 2016;28(9):1330-40.

47. Jacobs SR, Herman CE, Maclver NJ, Wofford JA, Wieman HL, Hammen $\mathrm{J}$, et al. Glucose uptake is limiting in T cell activation and requires CD28-mediated akt-dependent and independent pathways. J Immunol. 2008;180(7):4476-86.

48. Arase T, Uchida H, Kajitani T, Ono M, Tamaki K, Oda H, et al. The UDPglucose receptor P2RY 14 triggers innate mucosal immunity in the female reproductive tract by inducing IL-8. J Immunol. 2009;182(11):7074-84.

49. Norman AG, Miquel AG, Remy M. Short-chain fatty acids: ready for prime time? Nutr Clin Pract. 2006;21(4):351-66.

50. Al-Lahham SH, Peppelenbosch MP, Roelofsen H, Vonk RJ, Venema K. Biological effects of propionic acid in humans; metabolism, potential applications and underlying mechanisms. Biochim Biophys Acta-Mol Cell Biol L. 2010;1801(11):1175-83.

\section{Publisher's Note}

Springer Nature remains neutral with regard to jurisdictional claims in published maps and institutional affiliations. 